\title{
Sentence, proposition, and context: on the idea of an intermediate level
}

\author{
Riegelnik, Stefan
}

\begin{abstract}
In contemporary theories of language it is common to appeal to propositions as expressed by utterances of sentences. The aim of this paper is to question this idea, for as I argue, the relationship between sentences and propositions cannot be worked out in any rewarding way
\end{abstract}

Posted at the Zurich Open Repository and Archive, University of Zurich

ZORA URL: https://doi.org/10.5167/uzh-123518

Book Section

Published Version

Originally published at:

Riegelnik, Stefan (2014). Sentence, proposition, and context: on the idea of an intermediate level. In: Stalmaszczyk, Piotr. Semantics and Beyond: Philosophical and Linguistic Inquiries. Berlin: De Gruyter, 241-254. 


\title{
Stefan Riegelnik
}

University of Zurich

stefan.riegelnik@philos.uzh.ch

\section{Sentence, Proposition, and Context. On the Idea of an Intermediate Level}

\begin{abstract}
In contemporary theories of language it is common to appeal to propositions as expressed by utterances of sentences. The aim of this paper is to question this idea, for as I argue, the relationship between sentences and propositions cannot be worked out in any rewarding way.
\end{abstract}

Keywords: sentence, proposition, context, contextualism, minimalism, indexical expressions

\section{Introduction}

My question is: what is the relationship between a sentence and a proposition? This suggests, on the one hand, that there is such a relationship, and, on the other hand, that the relationship is substantial. In what follows, I want to dwell on this relationship, though I have to admit from the beginning that I have some doubts that the idea can be worked out in any rewarding way. Thus, the aim of this paper is to scrutinise potential ways to elucidate the problem and, by using the approach in question, give support to my doubts.

The question I want to investigate is connected with the discussion of what the primary bearers of truth are. Should we ascribe truth to sentences, propositions, utterances, statements, beliefs, or judgements? The list of potential candidates is long and each has its advocates and opponents. As interesting as this question seems to be, I have doubts that it can be answered in a way that is detached from any theory or framework. But it goes without saying that if one sticks with the idea that there is a relationship between a sentence and a proposition expressed by an utterance, then one considers propositions to be the object to which 
truth and falsity is ascribed in the first place. This calls for a clarification: nothing hinges on the terms sentence, proposition, etc. or on the phrase that a sentence expresses a proposition. The essence of this phrase seems to be the introduction of an intermediary level, or as Stalnaker puts it, an "extra step on the road from sentences to truth values" (Stalnaker 1970: 36). It is precisely this that propositions as falling apart from sentences come up to and it is exactly this idea the present paper will be concerned with.

I do not want to create an impression that neither the simile of an intermediary nor the question of how to spell it out is new or uncontroversial. David Lewis, for instance, criticises Stalnaker for giving a "misleading impression of simplicity" (Lewis 1980:39), for Stalnaker does not pay sufficient attention to the rules according to which we yield the content of a sentence relative to a context. However, in the end, he proclaims "solidarity forever" (Lewis 1980: 42). The idea of intermediaries appears in different guises and in different branches of philosophy. For instance, Tyler Burge in "Perceptual Entitlement" (2003) defends the idea of the existence of an intermediary between objects in the world and a subject having a belief about them. In contemporary theories of interpretation, many philosophers and linguists trace their approaches back to Frege's distinction between sense and reference. Although this is not Frege's line of reasoning, the introduction of such a distinction or one or more intermediaries might be justified by the use of a class of expression suggesting that the idea of such a second dimension would be required. The examples par excellence are so-called indexical expressions such as 'I', 'here', 'now', 'this', whose semantic value changes from context to context. From this it follows that the meaning of sentences containing these expressions - or in Fregean terminology, the thought they express - changes. The role of an intermediary such as a proposition then is to allow for a relativisation of sentences to contexts as expressing different propositions. Consider (1):

\section{(1) This is white.}

Depending on the reference of 'this', two utterances of the sentence (1) might express different propositions. From this example, it is easy to get 
to the conclusion that an intermediary level is not only innocuous, but required in order to grasp the variation. To put it generally, an intermediary level is supposed to wipe out all deficiencies of natural languages, which forestall obstinately a clear grasp of what is meant by an utterance of a sentence. This is achieved, in the case of an utterance of (1), simply by taking the semantic value of 'this' as the constituent of a (structured) proposition expressed by a sentence. The first impression is, admittedly, that this approach is innocuous, but this holds only if the fact that the interesting part just begins here is not neglected, by which I mean questions such as how names and indexical expressions are related to the objects they are supposed to refer to, how sentences or propositions are supposed to be true depending on the contributions of their parts, and if one holds that sentences and propositions stand apart - how they are related.

\section{Sentences and Propositions in Theory}

The construction of sentences and propositions and a relationship between them is inherent to theories that grant a decisive influence to an utterance's context. However, the crux of the matter lies within the details and poses a serious dilemma for these theories. The dilemma goes as follows: either the contextual influence is tied in some way to the sentence, which makes the appeal to propositions as distinctive entities vain, or, on the other hand, the role of sentences remains in the dark if the context contributes to the proposition independently, i.e. irrespective of the sentence which is supposed to express the proposition.

There might be good reasons to ascribe truth to propositions and not to sentences and hence to take the burden of explaining the transition from sentences to propositions. But since I have doubts that the debate can be settled in a general way, I will not discuss this question in isolation from theories appealing to this distinction. What I have claimed so far is that the idea of propositions as a paradigm for intermediacy is harmless if the relationship between sentences and propositions is explicated. One might object that it is the explanation of the use of expressions which fulfils the task of explaining how a proposition is expressed. But if so, 
then it is at least questionable to introduce intermediaries, for one can handle these questions without the ado of shady devices.

An alternative to an assessment of the relationship between sentences and propositions in isolation is a discussion of how such a framework might fit into a comprehensive theory of interpretation. This is suggested, too, by the fact that the classification of expressions and the use of technical terms such as sentence, proposition, context, etc. are already part and parcel of a comprehensive theory of interpretation, at least superficially. This shifts the discussion closer to actual theories of language which make use of frameworks built on the basis of different levels, thereby endowing each level with a particular explanatory function.

A further reason for examining the framework in the light of a particular theory is that if the terms sentence and proposition are not mere synonyms, a third factor is required which 'makes all the difference'. The third factor is supposed to explain the fact that different propositions are expressed by the same sentence. If there is no factor in respect of which different propositions are expressed, the difference between sentences and propositions is annihilated. But the appeal to this factor comes to nothing if the functioning of this factor remains a mere postulation and this functioning is to be explained by a theory of meaning.

Recently, a great deal of attention has been dedicated to the context of an utterance. I allude here to theories which dedicate ample room to the workings of contextual contributions for the determination of a proposition expressed by a sentence. These theories come in various grades of sophistication, but I will consider them in their simplest form. What is central to these theories is, in first place, a dismissive attitude towards traditional accounts of truth-conditional theories of interpretation. For the contention is that traditional theories neglect the role that the context of an utterance plays when it comes to explaining the meaning of utterances of the same sentence. From a different perspective, the notion of context, applied correctly, ought to perform the function of bridging the gap between mere sentences and the propositions expressed in a particular context. Irrespective of whether this kind of criticism is adequate, two positions on the extremes can be distinguished: the first, named literalism or minimalism, allows little or no contextual impact on the truth conditions of a sentence: contextual enrichment is limited to indexical 
expressions like 'I', 'here', 'now' or other 'triggers' at the logical level of a sentence. The opposite end of the spectrum is represented by contextualism: its governing thesis is that sentences are not truth evaluable unless they are completed and enriched by the context: truth conditions should be ascribed only to speech acts which are considered to be qua speech act in context. Despite the fierce debates and fundamental disagreements concerning the proper integration of contextual contributions in a comprehensive theory of interpretation, these theories are united by a commitment to a framework akin to the one discussed above. For instance, when presenting the tenets of their minimalism, Cappelen and Lepore maintain that

$[\ldots]$ the semantic content of a sentence $\mathrm{S}$ is the proposition that all utterances of $\mathrm{S}$ express. (Cappelen and Lepore 2005: 2)

Ironically, they explicitly refrain from explaining how such a (minimal) proposition is yielded. Although Cappelen's and Lepore's support for minimal propositions comes from different premises, this cannot free them from the question of how a minimal proposition is expressed or involved in the utterance of a sentence. That such an explanation is crucial is shown by the debate concerning the role of a minimal proposition if one and the same sentence is uttered both ironically and seriously. Putting the dubious notion of minimal proposition aside, what should be granted to minimalists is the restriction of contextual contributions to a set of expressions. One aspect of this is a taming of contextual impacts, but, and this seems to be more important here, they put the focus on what I might call the receptiveness of contextual contributions. I will address this point later in the paper.

Compared with minimalism, contextualism - be it in a radical or moderate version - is more liberal about the context contributing to a proposition expressed by a sentence. The core of these theories is that the context of utterance is, literally speaking, indispensable for the constitution of a proposition. As Recanati writes

[...] there is no level of meaning which is both (i) propositional (truthevaluable) and (ii) minimalist, that is, unaffected by top-down factors. (Recanati 2004: 90) 
Top-down factors are those contextual supplements which are not demanded by an expression, neither at the sentence's level nor within the hidden structure of a sentence. To put it counterfactually, without contributions of these kinds, no proposition is expressed, i.e. there is no truth value to assign. And it is a cardinal error, so the contextualist's story goes on, to ascribe a truth value to an entity which is not capable to be true or false. This line of criticism is substantiated by countless stories told in order to show how the context of utterance is supposed to influence the proposition expressed, or, in terms of truth-conditional semantics, how truth conditions vary with regard to contexts of utterance.

\section{Truth-conditional Semantics and Context}

What is often ignored in this context is the resonant question of what determines truth conditions. Shall we leave this to the context exclusively, as radical contextualists suggest? And if so, is the name 'truth conditional semantics' still appropriate or should 'context conditional semantics' be used instead? Be that as it may, the issue of the dependence of truth conditions seems to invoke a truism, for truth conditions are determined or constituted by whatever we say is true or false. And this, I will argue, poses a serious problem for the proponents of contextualism. In order to elucidate my point, I will adopt (2) as a basic scheme to state truth conditions:

(2) $s$ is true if and only if $p$

In (2), $p$ serves as a placeholder for the truth conditions of $s$. Or, to be precise, $p$ depends on whatever $s$ refers to. In the rudimentary classification presented above, $s$ either refers to a sentence (in the case of literalism or minimalism) or to a speech act (in the case of contextualism) which is qua definition in context and which expresses a proposition. Whatever the exact definition of the terms sentence, speech act, etc., it seems that Recanati is right in arguing that at least some sentences do not express any content or proposition at all, which comes down to the fact that they are not truth-evaluable. As the most obvious reasons for 
not ascribing a truth-value contextualists refer to indexical expressions and other expressions requiring contextual completion. Sentences containing one of these expressions are not truth-evaluable unless we know the reference and since we can only find out the reference in a particular context, i.e. if uttered at a particular time and place accompanied with the appropriate gestures, truth conditions are underdetermined unless enriched by the context. As Recanati puts it:

Indexical sentences possess a determinate (truth-evaluable) content only when uttered. Hence it is not obvious that such sentences, qua grammatical entities, possess content. (Recanati 2005: 172)

But Recanati appeals to the context as not only assigning a semantic value to a restricted range of expressions but also as a generic term for all kinds of 'fill-ins' which are alluded to in order to bridge the gap between linguistic meaning of a sentence and the meaning of an utterance or a speech act for which the term proposition is reserved. It follows from this that the framework Recanati appeals to requires a falling apart along the lines presented above. Generally, this is precisely the reason why contextualists have to appeal to a framework with intermediary levels. By integrating this in the scheme (2), one gets

(3) "the proposition expressed by $s$ relative to context $c$ " is true if and only if $p$.

Following this path, $s$ might refer to something like a semantic potential or a sentence-token which is, according to contextualism, not meaningful in isolation; i.e. only when uttered in a particular context. For this reason, it is incorporated into a meaningful complex as being suitable to be either true or false. Alternatively, $s$ in (2) refers to the entire complex on the left-hand side of the biconditional statement (3).

My initial question was how the assumed relationship between sentences and propositions might be worked out. This becomes even more important when considering that the context cannot add something to the right-hand side, viz. the truth conditions, which are not determined by $s$ or the complex on the right-hand side. To put it in a different way, if $p$ is determined entirely by the left part of a biconditional statement, the con- 
text does not have anything to add to $p$ which is not yet embodied in $s$ (or in the left part of the bi-conditional statement). On the other hand, the complex on the left-hand side cannot be incomplete, i.e. in need of contextual completion, for one of the central objections contextualists raise against traditional theories is that traditionalists assign a truth value to something which is not capable to be true or false. This, in turn, places the focus on the complex on the left part of (3) and on the question of how different propositions are expressed relative to different contexts.

A great deal of contextualists' turmoil stems from the countless stories told and comparisons of two utterances of the same sentence in different contexts. The aim of these stories is to show that the truth conditions of the sentence vary if some features of the story alter. One kind of example concerns the use of so-called gradable adjectives such as 'tall', 'hot', etc. It goes as follows: At first we are invited to imagine an utterance of a sentence, such as "Sarah is tall" in the context of a group of children. The utterance turns out to be true, for Sarah is growing fast compared to the other children in the group. Now imagine the same Sarah surrounded by a group of basketball players - the utterance of the sentence "Sarah is tall" now appears to be false, for Sarah is not tall compared to basketball players. Since Sarah does not shrink between both situations, contextualists conclude that the truth conditions of the sentence "Sarah is tall" are influenced by the context - otherwise we could not explain the change of the truth-value. The intention of telling these stories is to undermine the idea that truth conditions are determined by the (literal) meaning of an utterance of "Sarah is tall". And even if one disagrees with contextualists in respect of this line of criticism of traditional theories, one might still follow the contextualists' strategy, for it is hard to deny that at least sometimes the phrase "it depends on the context" is appropriate. The idea seems to be that both utterances share a sentence-type and that both utterances instantiate a token of that type. Such a token expresses a proposition relative to a context and the context becomes relevant for the determination of the proposition expressed in a yet to be determined way. Thus we can formulate biconditional statements such as: 
(4) The token of the sentence-type "Sarah is tall" relative to context $c 1$ expresses that the proposition $p$ is true if and only if Sarah is tall in context $c 1$.

The different contexts are supposed to explain that two utterances of the sentence-type "Sarah is tall" express different propositions. But how can this be spelled out?

A contextualist might argue that the proposition itself has nothing to do with the sentence uttered. This would simply mean that the contextualist denies the existence of a relationship between a sentence and the proposition. As odd as this might sound, it is not completely alien to contextualist thought. It comes down to the idea that the expressions used in an utterance are not necessarily involved in the proposition expressed. Some proponents of radical contextualism argue along these lines, for in contrast to proponents of moderate contextualism they hold that the context affects the proposition expressed even if there is no counterpart of it on the sentential level. As Recanati puts it:

Free enrichment is only one type of case in which a pragmatic process that is optional (in contrast to the contextual assignment of values to indexicals and free variables of all sorts) nevertheless affects the proposition expressed. (Recanati 2012: 71)

These contributions seem to represent a fourth level in the picture laid down, but this does not necessarily concern my question of how a proposition emerges from a sentence. On the other hand, any explanation of such a fourth factor remains problematic.

Another strategy to literally circumvent the issue might be to put the focus on the distinction between a sentence-type and its different tokens, which shifts propositions closer to sentence-tokens. However, in order to preserve the instantiation relation between a sentence-type and its tokens, the context cannot affect the sentence-tokens, for then they are no longer instances of the same sentence-type. One might argue that this is not so much a problem for advocates of contextualism, for they endorse a distinction between sentences and propositions, which does not bear on the distinction between types and tokens of sentences per se. This answer is not open to contextualists unless they state what the common 
features of utterances across different contexts are. In other words, contextualists have to retain both ideas - the type-token-distinction of sentences and the idea of contextual contributions to the proposition expressed. However, in order to do so, the sentence must be in some way open or receptive to contextual supplements. On the one hand, the typetoken-distinction requires an instantiation-relation, and on the other hand, the sentence must be in some way involved in the process of the determination of the proposition.

\section{Indexical Expressions and Contextual Supplements}

The class of indexical expressions seems to be predestined to satisfy both requirements. One 'part' of them remains fixed across contexts and the other 'part' varies, if used in an utterance, from context to context. This finds expression, most famously, in David Kaplan's distinction between content and character. According to his theory, the character of an indexical expression is a function which delivers, relative to a context, the content, i.e. the referee, of an indexical expression.

But it is obvious that the sentence "Sarah is tall" does not contain any of what normally counts as an indexical expression. The contextualist detour might be via the logical form of the sentence under discussion. We find this strategy, for instance, in Stanley (2005):

[...] all effects of extra-linguistic context on the truth-conditions of an assertion are traceable to logical form. (Stanley 2005: 33)

$[\ldots]$ the effects of context on the truth-conditional interpretation of an assertion are restricted to assigning values to elements in the expression uttered. Each such element brings with it rules governing what context can and cannot assign to it [...]. (Stanley 2005: 34)

The rationale for tracing contextual contributions back to the logical form of a sentence is that some contextualists see in unconstrained contextual contributions a threat to the principle of compositionality. In order to restrict them, they trace these contributions back to elements of the sentence. 
Placing explanatory weight on the logical level of a sentence involves the introduction of an additional level on the way to propositions. But even here 'counterparts' are required, both on the logical level and on the level of the sentence in order to explain the workings of all the contributions at all levels. The first task, then, is to reveal the 'real structure' of a sentence and to discover parts (hidden indexical expressions) or gaps at the logical level which ought to be filled or completed by the context.

In order to scrutinize this strategy, I take up the issue of Sarah's height. The context ought to provide the comparison class in relation to which Sarah is either tall or not. Following Stanley's strategy, the postulation of gaps or hidden indexical expressions places this version of contextualism closer to minimalism with the only difference that the class of indexical expressions is expanded. But what constitutes an indexical expression, be it on the surface or on the hidden level of a sentence? It is crucial to emphasise this point, for an element of a sentence cannot be simply a variable. For it runs counter to the idea that sentences represent a complex entity. Sentences do not carry variables - "Sara is tall relative to $\beta$ " just is not a sentence. This also holds for 'sentences' with gaps to be filled. Thus, the last alternative might well involve understanding these elements as indexical expressions. This is precisely what Stanley is claiming when he states that:

Broadly construed, an indexical is any contextual parameter, by which I mean any primitive expression whose denotation is supplied entirely by context, perhaps guided by a linguistic rule. (Stanley 2005: 38)

To specify an indexical expression in a narrow sense, Stanley draws on the 'method' used by minimalists - he simply makes a list:

In the narrow sense of "indexical", an indexical is a proper subset of context-sensitive expressions, one with the characteristics shared by words such as "I", "here", and "now", but not by "this", "that", "she", and "he". (Stanley 2005: 38)

However, I do not think that neither the broad nor the narrow understanding of indexical expressions is consistent. Firstly, if the group of 
indexical expressions is construed 'broadly', the question arises as to what distinguishes them from other singular terms? If the characteristic feature of indexical expressions is that the "denotation is supplied entirely by context", then almost every expression is of this kind.

With regard to the narrow conception of indexical expressions I want to point out that an explanation of the use of indexical expressions is more complex than suggests both by minimalists and moderate contextualists. Each indexical expression (or what normally counts as such) possesses distinctive features and we cannot declare an expression to be an indexical expression unless we specify these particular features. To explain this by an example: it is true that my utterance of the sentence "I am Stefan" does not contradict another person's utterance of the sentence "I am not Stefan". According to contextualism, the reason for this is that ' $\mathrm{I}$ ' is context-sensitive, i.e. the semantic value varies from context to context or from utterer to utterer. To put it generally, the context provides the semantic value of 'I' and we get biconditional statements like

(5) "I am Stefan" is true if and only if the speaker of the utterance "I am Stefan" is Stefan.

However, with such a paraphrase occurs a downgrade - the essential features of these expression get lost. Most prominently in the case of the first person pronoun, the immunity to error through misidentification is ignored. As Hans Sluga puts it:

But when we are sitting in an auditorium listening to a lecture and I whisper into your ear "The person who is now speaking is L.W.", I don't mean to be saying that I am Ludwig Wittgenstein and you are not likely to be in doubt about that. (Sluga 1996: 346f.)

A similar misidentification cannot happen in the case of 'I'. Let me put this differently; if contextualism were true, sentences such as "I am Stefan" would turn out to be identity statements if uttered by a person named 'Stefan'. It seems that contextualists have to swallow the pill and attempt a paraphrase, for otherwise the appeal to the context grasps at nothing. In turn, the appeal to indexical expressions as paradigms for the reality of context-sensitivity is also problematic. We neither have exact criteria for 
what indexical expressions are, while the supposed feature of contextsensitivity is insufficient to explain various uses of what typically counts as an indexical expression.

\section{Conclusion}

Shall we, nevertheless, retain the idea that the context of utterance is relevant in the sense that it determines the truth conditions? In particular, as by that we would retain the idea that sentences express proposition as well? For the problem with my argumentation might be that this line of reasoning throws out the baby with the bath water. The objection might be this: if we rule out the application of notions such as 'sentence', 'proposition' and 'context', we also give up the idea of revealing structures in natural language utterances. For it can hardly be denied that both utterances in the scenario have something in common. The question is, however, in virtue of what do both utterances have something in common? At any rate, the appeal to the context as being decisive for the proposition expressed seems to be highly dubious - at least if it is invoked to explain tensions resulting from repeating uses of expressions. And this makes propositions as a paradigm for intermediacy dubious as well.

\section{References}

Burge, Tyler 2003. Perceptual entitlement. Philosophy and Phenomenological Research 67, 503-548.

Cappelen, Herman and Ernest Lepore 2005. Insensitive Semantics: A Defense of Semantic Minimalism and Speech Act Pluralism. Malden, MA: Blackwell.

Lewis, David 1980. Index, context, and content. In: Papers in Philosophical Logic. Cambridge: Cambridge University Press. 1998, 21-44.

Preyer, Gerhard and Peter Georg (eds.) 2005. Contextualism in Philosophy: Knowledge, Meaning, and Truth. Oxford: Oxford University Press.

Recanati, François 2004. Literal Meaning. Cambridge: Cambridge University Press. Recanati, François 2005. Literalism and Contextualism: Some Varieties. In: G. Preyer and G. Peter (eds.), 171-196. 
Recanati, François 2012. Pragmatic Enrichment. In: G. Russell and D. Graff Fara (eds.) 67-78.

Russell, Gillian and Delia Graff Fara (eds.) 2012. Routledge Companion to Philosophy of Language. New York: Routledge.

Sluga, Hans 1996. 'Whose house is that?' Wittgenstein on the self. In: H. Sluga and D.G. Stern (eds.), 320-353.

Sluga, Hans and David G. Stern (eds.) 1996. The Cambridge Companion to Wittgenstein. Cambridge: Cambridge University Press.

Stalnaker, Robert. 1970. Pragmatics. In: Context and Content. Essays on Intentionality in Speech and Thought. New York: Oxford University Press, 31-47.

Stanley, Jason 2005. Language in Context: Selected Essays. Oxford: Oxford University Press. 